\title{
A clinical decision-making model for repeat surgical treatment of pectus Bar displacement: distance measurement after nuss procedure
}

Young Jo Sa ${ }^{1}$, Jongho Lee ${ }^{2}$, Jin Yong Jeong ${ }^{3^{*}}$, Moonhee Choi ${ }^{4}$, Soo Seog Park ${ }^{4}$, Sung Bo Sim ${ }^{5}$ and Keon Hyon Jo

\begin{abstract}
Background: Bar displacement is one of the most common and serious complications after the Nuss procedure. However, measurements of and factors affecting bar displacement have not been reported. The objectives of this study were to develop a decision model to guide surgeons considering repeat treatment and to estimate optimal cut-off values to determine whether reoperation to correct bar displacement is warranted.

Methods: From July 2011 to August 2013, ninety bars were inserted in 61 patients who underwent Nuss procedures for pectus excavatum. Group A did not need surgical intervention and Group B required reoperation for bar displacement. Bar position was measured as the distance from the posterior superior end of the sternal body to the upper border of the metal bar on lateral chest radiographs. The bar displacement index (BDI) was calculated using $D_{0}-D_{x} / D_{0} \times 100\left(D_{0}\right.$ : bar position the day after surgery; $D_{x}$ : minimal or maximal distance of bar position on the following postoperative days). The optimal cut-off values of BDI warranting reoperation were assessed on the basis of ROC curve analysis.

Results: Of the 61 patients, 32 had single bars inserted whereas 29 had parallel bars inserted. There was a significant difference in age ( $14.0 \pm 7.5$ vs. $23.3 \pm 12.0, p=0.0062)$, preoperative Haller index (HI) $(4.0 \pm 1.1$ vs. $5.0 \pm 1.0, p=0.033)$, and postoperative $\mathrm{HI}(2.7 \pm 0.4$ vs. $3.2 \pm 0.5 p=0.006)$ between the two groups. The optimal cut-off value of BDI was 8.7.

Conclusions: We developed a BDI model for surgeons considering performing reoperation after Nuss procedure. The optimal cut-off value of BDI was 8.7. This model may help surgeons to decide objectively whether corrective surgery should be performed. The main factors affecting the relationship between bar displacement and reoperation were age and preoperative $\mathrm{H}$.
\end{abstract}

Keywords: Pectus excavatum, Bar displacement, Minimally invasive surgery, Complication

\section{Background}

The Nuss procedure is used worldwide as a minimally invasive method to repair pectus excavatum (PE) [1]. Bar displacement is one of the most serious complications after the Nuss procedure. Incidence rates of bar displacement vary from 1.8 to $16.6 \%$ [1-5]. Bar displacement may result not only in morphologic changes

\footnotetext{
* Correspondence: jeong74@catholic.ac.kr

${ }^{3}$ Department of Thoracic and Cardiovascular Surgery, Incheon St. Mary's Hospital, College of Medicine, The Catholic University of Korea, 222 Banpo-daero, Seocho-gu, Seoul 137-701, Republic of Korea

Full list of author information is available at the end of the article
}

of the thorax, but can also result in life threatening conditions that require emergency surgery [6]. It occurs most frequently within 30 days following surgery.

Bar displacement can occur through the following three mechanisms: bar flipping, lateral sliding, and hinge point disruption [7]. Several techniques have been applied to prevent bar displacement, including the use of stabilizers, third point of fixation, bar fixation with stainless-steel wire, using a shorter bar, multipoint pericostal fixation, and placement of two bars [7-11].

Displacement of the pectus bar after surgery can be mild to severe. Some authors have measured the degree 
of bar displacement as the slope angle of the bar position and classified bar displacement as excellent, incomplete, or poor $[10,12]$. Bar displacement requiring reoperation is also referred to as " $90^{\circ}$ or $180^{\circ}$ rotation," "major displacement," and "significant displacement" [2, 4, 13].

However, measurements of and factors affecting bar displacement have not been examined rigorously. The objectives of this study were to develop a decision model to guide surgeons considering repeat treatment to correct bar displacement and to estimate optimal cut-off values when considering reoperation using receiver operating characteristic (ROC) curves.

\section{Methods}

\section{Study sample}

From July 2011 to August 2013, ninety bars were inserted in 61 patients who underwent Nuss procedures for the treatment of PE. Indications for surgery were: clinical symptoms such as exertional dyspnea, chest discomfort, and growth retardation; Haller index $(\mathrm{HI})>$ 3.25; cardiac deformity compressed by depressed chest wall; and psychological problems. We divided the patients into two groups: Group A (Table 1: category 1,2) who did not need more surgical intervention; and Group B (Table 1: category 3, 4) who required reoperation for bar displacement. Written informed consent was obtained from each patient prior to surgery. The study was approved by the Institutional Review Board of Incheon St. Mary's Hospital, College of Medicine, the Catholic University of Korea. All procedures were carried out by one surgeon.

\section{Surgical technique}

Under general anesthesia with a single endotracheal tube intubation, the patient was placed in a supine position with both arms abducted. The pectus bar size was measured as the distance around the anterior chest wall from one side to the other side of the chest wall on the

Table 1 Grades of pectus bar displacement

\begin{tabular}{ll}
\hline Categories & Description \\
\hline 1 (Mild) & $\begin{array}{l}\text { There is no displacement of the bar. } \\
\text { Or } \\
\text { Displacement of the bar has occurred but there } \\
\text { are no external morphologic changes. }\end{array}$ \\
2 (Moderate) & $\begin{array}{l}\text { Displacement of the bar and external morphologic } \\
\text { changes have occurred but correction of the bar } \\
\text { displacement is not necessary. }\end{array}$ \\
3 (Severe) & $\begin{array}{l}\text { Displacement of the bar and external morphologic } \\
\text { changes have occurred and correction of the bar } \\
\text { displacement is necessary. }\end{array}$ \\
$\begin{array}{l}\text { Displacement of the bar has occurred and resulted } \\
\text { in compression of the intrathoracic organs, high risk } \\
\text { of organ injury, or abnormal EKG. Urgent correction } \\
\text { of the bar displacement should be performed. }\end{array}$ \\
\hline
\end{tabular}

midaxillary line. The bar was bent according to the morphologic classification of $\mathrm{PE}$ and the crane technique was routinely applied [11]. Each tiny skin incision was made on both lateral chest walls. A subcutaneous tunnel and hinge point were made. A thoracoscopic port (MiniPort $2 \mathrm{~mm}$, Tyco Healthcare UK Ltd., Gosport, UK) for a needlescope (2-mm mini fiber telescope, Richard Wolf GmbH, Knittlingen, Germany) was applied cranially from the skin incision along the midaxillary line with $\mathrm{CO}_{2}$ insufflation.

The pectus clamp was introduced through the hinge point. Another $2 \mathrm{~mm}$ port for endoscissors was inserted through a tiny skin incision (Richard Wolf $\mathrm{GmbH}$ ). After completing dissection of the substernal space with endoscissors under direct vision using video assisted thoracoscopy [14], a $20 \mathrm{Fr}$. chest tube (Argyle thoracic PVC catheter, Covidien llc, Mansfield, MA, USA) was inserted through both hinge points following the pectus clamp. The pectus bar was inserted under the guidance of the chest tube and rotated. After reassessing the external morphology of the anterior chest wall, anterior pericostal fixation of the bar was performed with No. 5 Ethibond (Ethibond Excel, Ethicon Inc., Somerville, NJ, USA) using an endo-needleholder (Olympus Winter \& Ibe GmbH, Hamburg, Germany) or Deschamps needle (B. Braun Aesculap AG, Tuttlingen, Germany) under video assisted thoracoscopy [15]. Both lateral fixations were made with fixators. Drainage catheters were inserted into the pleural cavity of one side and the soft tissue layer of the other side if the patient was a child or into the pleural cavities of both sides if the patient was an adult for draining gas and blood.

On the day of surgery, radiologic studies were done several times in the operating room, recovery room, and intensive care unit for the early detection of major complications such as hemothorax, tension pneumothorax, and major bar displacement. Serial chest PA and lateral views were taken daily from the next day onward. The pectus bar was removed 2 years after insertion for patients less than 12 years old, and 2.5 years after insertion for patients between 12 and 18 years old. For patients over 18 years old, the bar was removed 3 years after insertion.

\section{Measurement of bar displacement}

Bar position was measured as the distance from the posterior superior end of the sternal body to the upper border of the metal bar on the lateral chest radiograph (Fig. 1). The bar displacement distance was obtained by measuring bar position the day after surgery and the next postoperative days ( 1 week, 2 weeks, 1 month, and 3 months). The categories of bar displacement were defined as in Table 1 and Fig. 2. 


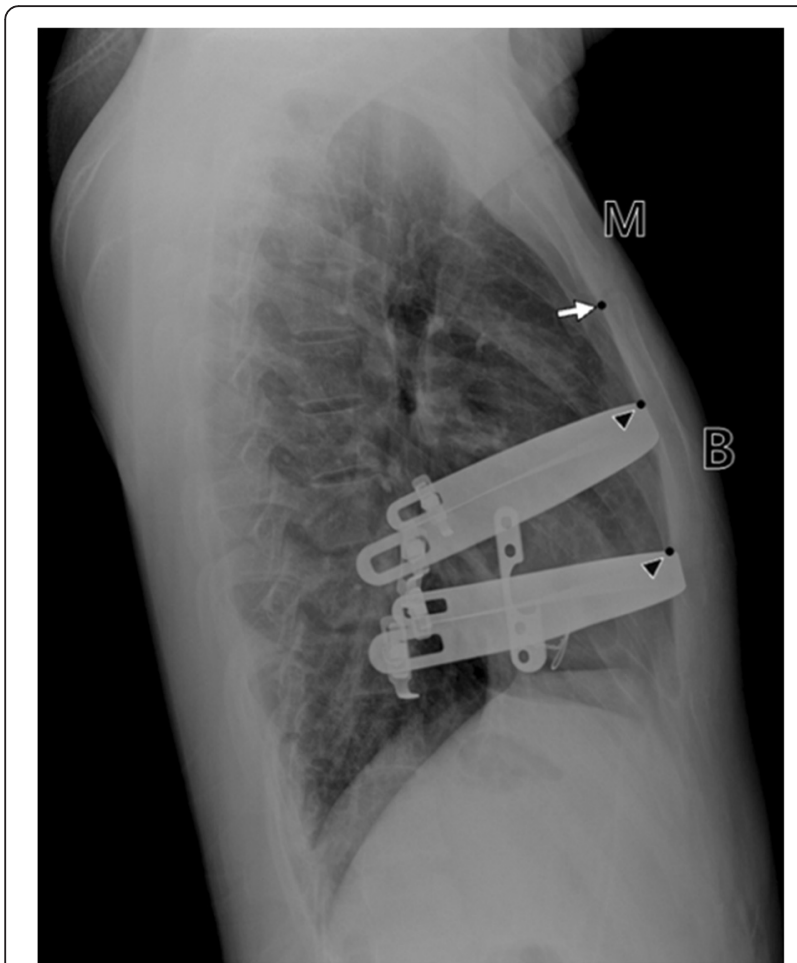

Fig. 1 Measurement of bar position. M: manubrium; B: body of the sternum; White arrow: point on the posterior superior end of the sternal body; Black arrow head: point on the superior border of the metal bar

The bar displacement index (BDI) was calculated using the formula of $D_{0}-D_{x} / D_{0} \times 100$, where $D_{0}$ is the bar position the day after surgery, and $D_{x}$ is the minimal or maximal bar position the next postoperative days. In addition, (+) represented upward displacement of the bar, whereas (-) represented downward displacement of the bar.

\section{Statistical analysis}

Continuous and categorical variables were expressed as means $\pm \mathrm{SD}$ and percentages, respectively. Differences between patients in Group A and Group B were analyzed by Fisher's exact test for categorical data, and unpaired $t$ test for continuous parametric data. Differences between patients in each bar-displacement tertile were assessed by ANOVA or $\chi^{2}$ tests. Bivariate correlation analysis was utilized to examine the associations between two variables. Statistical significance was defined as a $p$ value less than 0.05 .

The usefulness of BDI for predicting reoperation was assessed by receiver operating characteristic (ROC) curve analysis. The cut-off values of BDI were defined on the basis of ROC curve analysis by identifying the values of BDI that gave the best combination of sensitivity and specificity. All analyses were performed with SAS 9.3 software.

\section{Results}

\section{Patient characteristics}

Baseline characteristics of patients are summarized in Table 2. There were 7 women and 54 men with a mean age of 15.1 years (range, 3 to 40 years). Of the 61 patients, 32 had single bars inserted, whereas 29 had parallel bars inserted. Of the 90 bars, 42 moved upward postoperatively, 27 moved downward, and 21 moved both upward and downward.

There were no significant differences in sex, BMI (height and weight), pectus excavatum type, or the number of bar insertions between Groups A and B (Table 2).

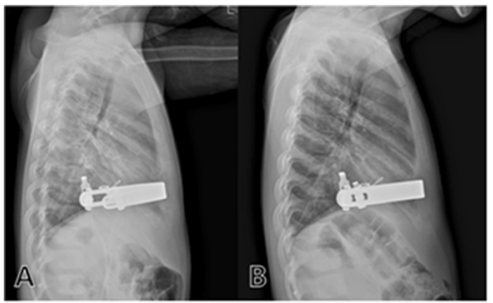

Category 1

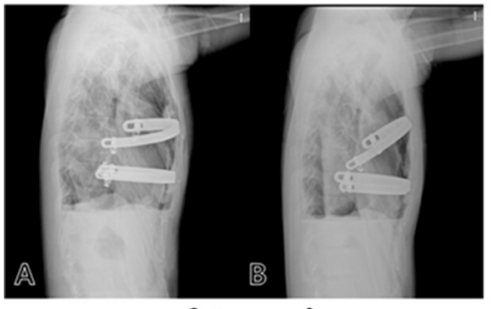

Category 3

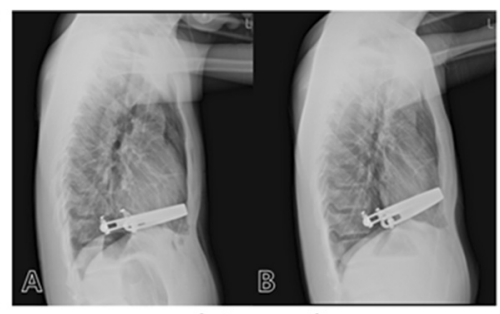

Category 2

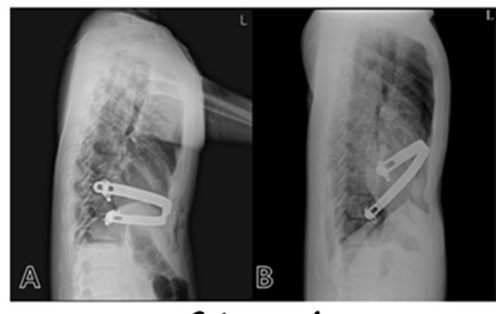

Category 4

Fig. 2 Radiographs describing the four categories of bar displacement. a preoperative; b: postoperative 
Table 2 Patient characteristics and univariate analysis of clinical factors associated with bar displacement

\begin{tabular}{lllll}
\hline Variable & $\begin{array}{l}\text { All } \\
(N=61)\end{array}$ & $\begin{array}{l}\text { Group A } \\
(N=54)\end{array}$ & $\begin{array}{l}\text { Group B } \\
(N=7)\end{array}$ & $P$ \\
\hline Age & $15.1+8.6$ & $14.0+7.5$ & $23.3 \pm 12.0$ & 0.0062 \\
Sex & & & & 0.5856 \\
$\quad$ Male & 54 & 47 & 7 & \\
$\quad$ Female & 7 & 7 & 0 & \\
BMl & $18.4+2.8$ & $18.2 \pm 2.8$ & $19.5 \pm 2.4$ & 0.2582 \\
Height & $1.5+0.3$ & $1.5 \pm 0.3$ & $1.6 \pm 0.3$ & 0.4543 \\
$\quad$ Weight & $46.8+18.6$ & $46.0 \pm 18.9$ & $53.3 \pm 16.4$ & 0.3361 \\
HI & & & & \\
$\quad$ Pre- HI & $4.1+12$ & $4.0 \pm 1.1$ & $5.0 \pm 1.0$ & 0.033 \\
Post- HI & $2.7+0.4$ & $2.7 \pm 0.4$ & $3.2 \pm 0.5$ & 0.0006 \\
$\quad$ Diff- HI & $1.4+1.1$ & $1.3 \pm 1.1$ & $1.7 \pm 1.0$ & 0.3175 \\
Type & & & & 0.4288 \\
Symmetric & 32 & 27 & 5 & \\
Asymmetric & 29 & 27 & 2 & \\
No. of inserted bar & & & & \\
1 & 32 & 30 & 2 & \\
2 & 29 & 24 & 5 & \\
\hline
\end{tabular}

There were significant differences in age (14.0 \pm 7.5 vs. $23.3 \pm 12.0, p=0.0062)$, preoperative HI $(4.0 \pm 1.1$ vs. $5.0 \pm 1.0, p=0.033)$, and postoperative HI $(2.7 \pm 0.4$ vs. $3.2 \pm 0.5 p=0.006)$ between the two groups. However, $\mathrm{HI}$ difference (postoperative $\mathrm{HI}$ - preoperative HI) was similar between groups.

\section{ROC curve analysis of BDI for detecting bar displacement}

Clinical characteristics of patients stratified by bar displacement index tertiles are summarized in Table 3. The three tertile groups were comparable in age, sex, pre-HI, post-HI, and type. However, there were significant differences in BMI (weight and height), the number of inserted bars, and grade among the three tertile groups. Patients with higher displacement indexes (tertile 3) were taller and heavier with higher $\mathrm{BMI}$, grade, and number of inserted bars than those in tertile $1(p<0.05)$.

ROC curve analysis was used to assess bar displacement to determine whether surgical repair was necessary. The optimal cut off value of BDI was 8.7. The area under the ROC curve was 0.858 (95\% CI, 0.769-0.923; $p<0.0001$, Fig. 3).

Table 3 Basic characteristics of bar displacement index tertiles

\begin{tabular}{|c|c|c|c|c|}
\hline \multirow[t]{2}{*}{ Variable } & \multicolumn{4}{|l|}{ |(bar0-bar1)/bar0 | x 100} \\
\hline & Tertile $1(0-2.9) N^{*}=30$ & Tertile $2(2.9-7.2) N^{*}=30$ & Tertile $3(>7.2) N^{*}=30$ & $P$ \\
\hline Age & $14.5 \pm 9$ & $15.5 \pm 7.6$ & $18.7 \pm 7.5$ & 0.1167 \\
\hline Sex & & & & 0.6644 \\
\hline Male & 22 & 24 & 21 & \\
\hline Female & 8 & 6 & 9 & \\
\hline $\mathrm{BMI}$ & $18 \pm 2.7$ & $18.1 \pm 2.9$ & $19.9 \pm 2.6$ & 0.0135 \\
\hline Height & $1.5 \pm 0.3$ & $1.6 \pm 0.2$ & $1.7 \pm 0.2$ & 0.0141 \\
\hline Weight & $43.6 \pm 19.4$ & $48.7 \pm 16.4$ & $57.9 \pm 13.7$ & 0.0049 \\
\hline Pre-HI & $4 \pm 1.3$ & $4.1 \pm 1$ & $4.4 \pm 1.2$ & 0.3708 \\
\hline Post-HI & $2.6 \pm 0.3$ & $2.8 \pm 0.4$ & $2.8 \pm 0.4$ & 0.0725 \\
\hline Type & & & & 0.8747 \\
\hline Symmetric & $16(53.3 \%)$ & $15(50 \%)$ & $17(56.7)$ & \\
\hline Asymmetric & $14(46.7 \%)$ & $15(50 \%)$ & 13(43.3) & \\
\hline Grade & & & & $<.0001$ \\
\hline 1 & $30(100 \%)$ & $25(83.3 \%)$ & 13(43.3\%) & \\
\hline 2 & 0 & $3(10 \%)$ & $9(30 \%)$ & \\
\hline 3 & 0 & $2(6.7 \%)$ & $5(16.7 \%)$ & \\
\hline 4 & 0 & 0 & $3(10 \%)$ & \\
\hline No. of inserted bars & & & & $<.0 .001$ \\
\hline 1 & $19(63.3 \%)$ & 10(33.3\%) & $3(10 \%)$ & \\
\hline 2 & $11(36.7 \%)$ & $20(66.7 \%)$ & $27(90 \%)$ & \\
\hline
\end{tabular}

$N^{*}$ number of bar 


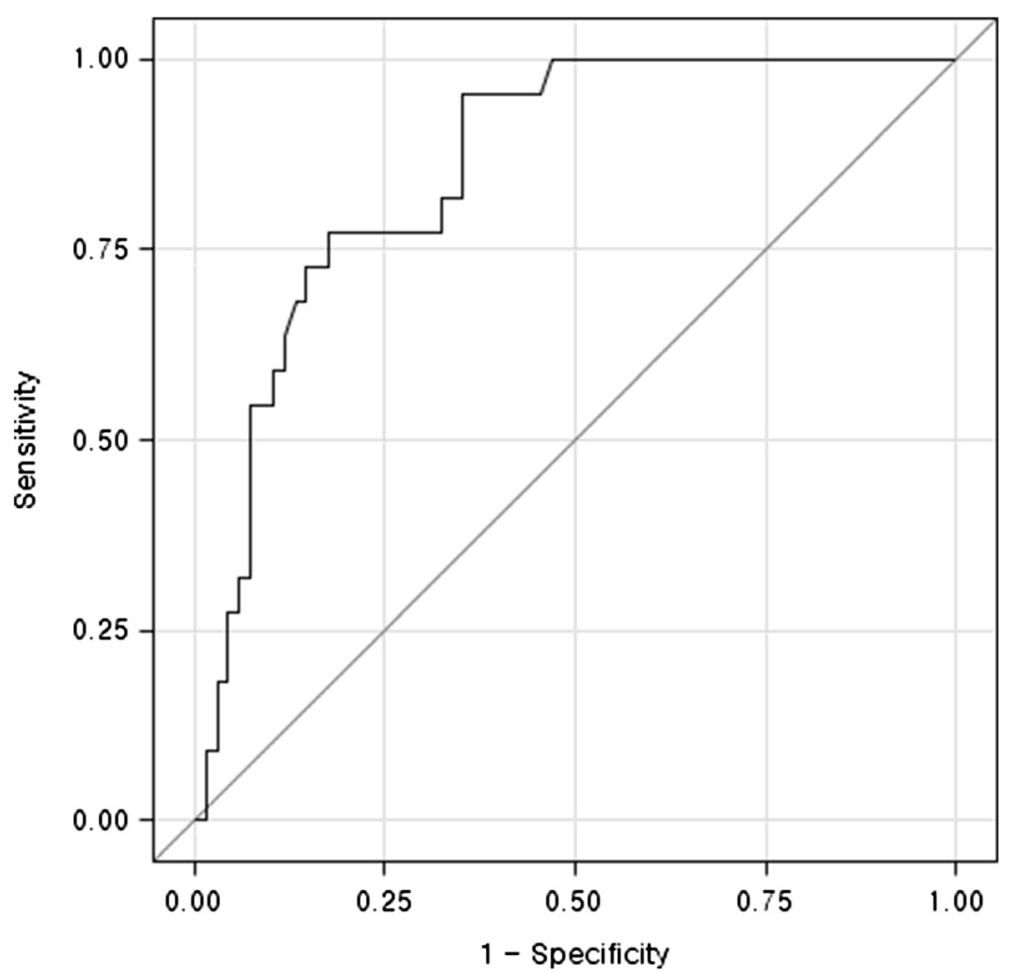

Fig. 3 The ROC curve of the bar displacement index for the detection of bar displacement. The area under the curve was $0.858(95 \% \mathrm{Cl}, 0.769-0.923)$. The cut-off value of BDI was 8.7

\section{Discussion}

Park et al. [7] described a variety of mechanisms governing bar displacement, including flipping along the axis of the hinge, sliding laterally to the left or right side, and backward shifting due to stripping of the intercostal musculature. Flipping of the bar is the most common type of displacement $[2,4,13]$. Classifications of bar positions differ between authors. Bar flipping displacement requiring correction is presented in abstract terms such as " $90^{\circ}$ or $180^{\circ}$ rotation," "major displacement," and "significant displacement" $[2,4,13]$. Others defined bar positions as excellent (the bar facing the sternum at right angles, less than $15^{\circ}$ ), incomplete (minimal bar displacement of less than $45^{\circ}$, resulting in minor depression of the sternum), and poor $\left(90^{\circ}\right.$ flipped bar with recurred sternal depression) [10, 12].

Displacement of the pectus bar after surgery can be mild to severe. Some authors measure the degree of bar displacement as the slope angle of the bar position. Because these measurements are either obscure or complex, we simply measured the pectus bar position on the lateral projections of chest X-rays (Fig. 1). We defined the BDI as the ratio of the maximal difference between the displacement distance/the distance of the bar position on the day after surgery. ROC curve analysis showed that the cut-off value of BDI indicating a need for corrective surgery for bar displacement was 8.7. Of
61 patients, $46(75.4 \%)$ and $8(13.1 \%)$ patients were grades 1 and 2, respectively. These patients did not need surgical correction. Of the remainder (4 patients in grade 3 and 3 patients in grade 4), 5 patients (8.2\%) underwent repeat surgery for correction. Of 4 patients in grade 3, two patients who were satisfied with the external morphological results did not want to undergo reoperation. When the slope angle of the bar was measured in 5 patients with grades 3 and 4 who underwent corrective reoperation, they had bar displacements with angles over $30^{\circ}$ (major displacement) [4].

Several devices and techniques have been used to prevent bar displacement [8-11]. We performed not only lateral fixation of the bar, but also anterior pericostal fixation under video-assisted thoracoscopy and the crane technique. This procedure showed a low rate of reoperation to corrent displacement of the pectus bar [16]. Thoracoscopy can display the deepest point of the anterior chest wall during the bar fixation procedure and the position of the bar after completing the procedure, which can reduce the rate of bar displacement as previously mentioned [2]. It also enables safe dissection of the substernal space and identification of the bleeding point and lung entrapment by the bar. However, the application of rigid thoracoscopy may have a disadvantage when showing the opposite side beyond the deepest point in severe pectus excavatum. This problem can be 
overcome by elevating the depressed anterior chest wall with the use of devices such as the crane technique, suction cups, and sternal lifts [11, 17-19]. Elevating the depressed sternum increases the substernal space, which not only improves the thoracoscopic visual field, but also eliminates the risk of cardiac injury by the dissector, therefore allowing less traumatic placement of the pectus bar.

\section{Conclusions}

We developed a BDI model to help surgeons decide objectively whether reoperation is necessary after the Nuss procedure. The main factors indicating that displaced bars require reoperation are age and preoperative HI.

\section{Consent}

Written informed consent was obtained from all patients or their parents for the publication of this report and any accompanying images.

\section{Competing interests}

The authors declare that they have no competing interests.

\section{Authors' contributions}

YJS: Study design, data collection and manuscript writing. JL: Study design and manuscript writing. JYJ: Study design, data collection, statistical analysis and interpretation, and manuscript writing. MC: Data collection. SSP: Study design and data collection. SBS: Study design and manuscript writing. KHJ: Study design and data collection. All authors read and approved the final manuscript.

\section{Acknowledgments}

The authors would like to thank Kyungdo Han, Department of Biostatistics, The Catholic University of Korea, for contributing to the statistical analysis of the data.

\section{Author details}

'Department of Thoracic and Cardiovascular Surgery, Yeouido St. Mary's Hospital, College of Medicine, The Catholic University of Korea, Seoul, Republic of Korea. ${ }^{2}$ Department of Thoracic and Cardiovascular Surgery, Daejeon St. Mary's Hospital, College of Medicine, The Catholic University of Korea, Seoul, Republic of Korea. ${ }^{3}$ Department of Thoracic and Cardiovascular Surgery, Incheon St. Mary's Hospital, College of Medicine, The Catholic University of Korea, 222 Banpo-daero, Seocho-gu, Seoul 137-701, Republic of Korea. ${ }^{4}$ Department of Anesthesiology, Incheon St. Mary's Hospital, College of Medicine, The Catholic University of Korea, Seoul, Republic of Korea. ${ }^{5}$ Department of Thoracic and Cardiovascular Surgery, St. Paul's Hospital, College of Medicine, The Catholic University of Korea, Seoul, Republic of Korea. ${ }^{6}$ Department of Thoracic and Cardiovascular Surgery, Seoul St. Mary's Hospital, College of Medicine, The Catholic University of Korea, Seoul, Republic of Korea.

\section{Received: 13 August 2015 Accepted: 13 January 2016}

Published online: 19 January 2016

\section{References}

1. Nuss D, Kelly Jr RE, Croitoru DP, Katz ME. A 10-year review of a minimally invasive technique for the correction of pectus excavatum. J Pediatr Surg. 1998;33:545-52.

2. Hebra A, Swoveland B, Eqbert M, Tagge EP, Georgeson K, Othersen Jr HB, et al. Outcome analysis of minimally invasive repair of pecuts excavatum: review of 251 cases. J Pediatr Surg. 2000;35:252-8.

3. Tedde ML, Campos JR, Das-Neves-Pereira JC, Abrāo FC, Jatene FB. The search for stability: bar displacement in three series of pectus excavatum patients treated with the Nuss technique. Clinics (Sao Paulo). 2011;66:1743-6.
4. Park HJ, Lee SY, Lee CS, Youm W, Lee KR. The Nuss procedure for pectus excavatum: evolution of techniques and early results on 322 patients. Ann Thorac Surg. 2004;77:289-95.

5. Pilegaard HK, Licht PB. Early results following the Nuss operation for pecuts excavatum - a single-institution experience of 383 patients. Interact Cardiovasc Thorac Surg. 2008;7:54-7.

6. Hoel TN, Rein KA, Svennevig JL. A life-threatening complication of the Nussprocedure for pectus excavatum. Ann Thorac Surg. 2006:81:370-2

7. Park HJ, Chung WJ, Lee IS, Kim KT. Mechanism of bar displacement and corresponding bar fixation techniques in minimally invasive repair of pecuts excavatum. J Pediatr Surg. 2008:43:74-8.

8. Nuss D, Croitoru DP, Kelly Jr RE, Goretsky MJ, Nuss KJ, Gustin TS. Review and discussion of the complications of minimally invasive pecuts excavatum repair. Eur J Pediatr Surg. 2002:12:230-4.

9. Hebra A, Gauderer MW, Tagge EP, Adamson WT, Othersen Jr HB. A simple technique for preventing bar displacement with the Nuss repair of pecuts excavatum. J Pediatr Surg. 2001;36:1266-8.

10. Uemura S, Nakagawa Y, Yoshida A, Choda Y. Experience in 100 cases with the Nuss procedure using a technique for stabilization of the pectus bar. Pediatr Surg Int. 2003;19:186-9.

11. Park HJ, Jeong JY, Jo WM, Shin JS, Lee IS, Kim KT, et al. Minimally invasive repair of pectus excavatum: a novel morphology-tailored, patient-specific approach. J Thorac Cardiovasc Surg. 2010;139:379-86.

12. Del Frari B, Schwabegger AH. How to avoid pectus bar displacement in the MIRPE or MOVARPE technique: results of 12 years' experience. Ann Plast Surg. 2014;72:75-9.

13. Castellani C, Schalamon J, Saxena AK, Höellwarth ME. Early complications of the Nuss procedure for pecuts excavatum: a prospective study. Pediatr Surg Int. 2008;24:659-66.

14. Jeong JY, Lee J. Use of needlescope and crane technique to avoid cardiac injury in Nuss procedure. Ann Thorac Surg. 2014;98:386-7.

15. Jeong JY, Lee J. Needlescope-assisted 3-point fixation of the pectus bar in the Nuss procedure. J Thorac Cardiovasc Surg. 2014;147:1721-2.

16. Yoo G, Rha EY, Jeong JY, Lee J, Sim SB, Jo KH. Emerging fixation technique to prevent pectus bar displacement: needlescope-assisted 3-point fixation. Thorac Cardiovasc Surg. 2015. DOI: 10.1055/s-0035-1556818

17. Schier F, Mahr M, Klobe E. The vacuum chest wall lifter: an innovative, nonsurgical addition to the management of pectus excavatum. J Pediatr Surg. 2005;40:496-500

18. Tedde ML, de Campos JR, Wihlm JM, Jatene FB. The Nuss procedure made safer: an effective and simple sternal elevation manoeuvre. Eur J Cardiothorac Surg. 2012;42:890-1.

19. Johnson WR, Fedor D, Singhal S. A novel approach to eliminate cardiac perforation in the nuss procedure. Ann Thorac Surg. 2013:95:1109-11.

Submit your next manuscript to BioMed Central and we will help you at every step:

- We accept pre-submission inquiries

- Our selector tool helps you to find the most relevant journal

- We provide round the clock customer support

- Convenient online submission

- Thorough peer review

- Inclusion in PubMed and all major indexing services

- Maximum visibility for your research

Submit your manuscript at www.biomedcentral.com/submit
) BioMed Central 